\title{
Etapas ac-ac directas con un número reducido de dispositivos operando en modo discontínuo
}

\author{
H. Sarnago, Ó. Lucía, A. Mediano y J.M. Burdío. \\ Grupo de Electrónica de Potencia y Microelectrónica (GEPM) \\ Instituto de Investigación en Ingeniería de Aragón (I3A) \\ Universidad de Zaragoza, Mariano Esquillor s/n, 50018, Zaragoza, Spain. \\ Tel. +34-976762707, Fax +34-976762043, e-mail: hsarnago@unizar.es
}

\begin{abstract}
El calentamiento doméstico por inducción se basa en generar el calor directamente en el recipente mediante la aplicación de un campo magnético variable de alta frecuencia. Dada la potencia requerida, sistemas de potencia a eleveda frecuencia son empleados. Los esquemas tradicionales emplean dos grandes bloques: una etapa previa rectificadora, encargada de generar un bus de tensión contínua con el que alimentar a la segunda de las etapas, la inversora. Ésta es la encargada de generar la corriente de alta frecuencia con la que alimentar el inductor. Esquemas clásicos emplean topologías de dos dispositivos como el semi-puente resonante serie, dada la potencia alcanzada, sin embargo, etapas de un solo dispositivo han sido también empleadas con el fin de reducir costes, elevando la complejidad de control del conjunto. En esta ponencia se expone un nuevo concepto: la unión de ambas etapas en un conjunto, lo que se denomina como etapas directas ac-ac, puesto que no existe una conversión intermedia a un bus de contínua. La etapa conjunta se basa en la topología 1SWZVS en modo cicloconvertidor con dos elementos unipolares y bidireccionales en serie, logrando un dispositivo bipolar y bidireccional, que gobierna el sistema. Adicionalmente, se ha propuesto un modo de operación discontínuo que permite incrementar la eficiencia del conjunto para potencias elevadas.
\end{abstract}

